\title{
Mutual exclusion of Asaia and Wolbachia in the reproductive organs of mosquito vectors
}

Paolo Rossi ${ }^{1 \dagger}$, Irene Ricci ${ }^{1 \dagger}$, Alessia Cappelli ${ }^{1}$, Claudia Damiani ${ }^{1}$, Ulisse Ulissi ${ }^{1}$, Maria Vittoria Mancini ${ }^{1}$, Matteo Valzano ${ }^{1}$, Aida Capone', Sara Epis ${ }^{2}$, Elena Crotti ${ }^{3}$, Bessem Chouaia ${ }^{3}$, Patrizia Scuppa ${ }^{1}$, Deepak Joshi ${ }^{4}$, Zhiyong $\mathrm{Xi}^{4}$, Mauro Mandrioli ${ }^{5}$, Luciano Sacchi ${ }^{6}$, Scott L. O'Neill' ${ }^{7}$ and Guido Favia ${ }^{{ }^{*}}$

\begin{abstract}
Background: Wolbachia is a group of intracellular maternally inherited bacteria infecting a high number of arthropod species. Their presence in different mosquito species has been largely described, but Aedes aegypti, the main vector of Dengue virus, has never been found naturally infected by Wolbachia. Similarly, malaria vectors and other anophelines are normally negative to Wolbachia, with the exception of an African population where these bacteria have recently been detected. Asaia is an acetic acid bacterium stably associated with several mosquito species, found as a dominant microorganism of the mosquito microbiota. Asaia has been described in gut, salivary glands and in reproductive organs of adult mosquitoes in Ae. aegypti and in anophelines. It has recently been shown that Asaia may impede vertical transmission of Wolbachia in Anopheles mosquitoes. Here we present an experimental study, aimed at determining whether there is a negative interference between Asaia and Wolbachia, for the gonad niche in mosquitoes.
\end{abstract}

Methods: Different methods (PCR and qPCR, monoclonal antibody staining and FISH) have been used to address the question of the co-localization and the relative presence/abundance of the two symbionts. PCR and qPCR were performed to qualitatively and quantitatively verify the distribution of Asaia and Wolbachia in different mosquito species/organs. Monoclonal antibody staining and FISH were performed to localize the symbionts in different mosquito species.

Results: Here we provide evidence that, in Anopheles and in other mosquitoes, there is a reciprocal negative interference between Asaia and Wolbachia symbionts, in terms of the colonization of the gonads. In particular, we have shown that in some mosquito species the presence of one of the symbionts prevented the establishment of the second, while in other systems the symbionts were co-localized, although at reduced densities.

Conclusions: A mutual exclusion or a competition between Asaia and Wolbachia may contribute to explain the inability of Wolbachia to colonize the female reproductive organs of anophelines, inhibiting its vertical transmission and explaining the absence of Wolbachia infection in Ae. aegypti and in the majority of natural populations of Anopheles mosquitoes.

Keywords: Asaia, Competition, Mosquito, Wolbachia

\footnotetext{
* Correspondence: guido.favia@unicam.it

${ }^{\dagger}$ Equal contributors

'Scuola di Bioscienze e Medicina Veterinaria, Università di Camerino, 62032

Camerino, MC, Italy

Full list of author information is available at the end of the article
}

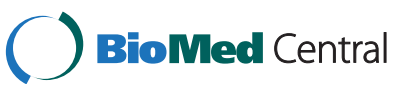

(c) 2015 Rossi et al.; licensee BioMed Central. This is an Open Access article distributed under the terms of the Creative Commons Attribution License (http://creativecommons.org/licenses/by/4.0), which permits unrestricted use, distribution, and reproduction in any medium, provided the original work is properly credited. The Creative Commons Public Domain Dedication waiver (http://creativecommons.org/publicdomain/zero/1.0/) applies to the data made available in this article, unless otherwise stated. 


\section{Background}

Most insects harbour mutualistic microorganisms that provide specific traits to the host, conferring evolutionary/adaptive advantages. Many beneficial symbionts are acquired by environmental uptakes in every generation, while few are located in the reproductive organs. As a consequence of this localization, they can easily be acquired by the offspring to ensure their transmission to the next insect generations by vertical transmission $[1,2]$. To date, only two bacteria have been shown to be located in the reproductive organs in several mosquito species: the alfa-proteobacteria Wolbachia and Asaia [3].

Wolbachia is a group of obligate intracellular bacteria that infect arthropods and nematodes [4]. Within arthropods, Wolbachia infects a wide spectrum of insects, reflecting the ability of these bacteria to manipulate host reproduction, favouring their own maternal transmission [5].

These intracellular bacteria were firstly described in Culex pipiens [6], but more recently they have been detected in mosquitoes from several genera, including Aedes, Culex, Coquillettidia, and Mansonia. Interestingly, until very recently, Wolbachia has not been recorded to naturally infect representatives of the genus Anopheles, that comprises more than 300 different species, about 60 of whose are malaria vectors [7]. Only very recently, the presence of Wolbachia has been detected in a few individuals of a natural small population of Anopheles gambiae. Interestingly, the positive mosquito individuals detected in this study clustered into only a few of the examined mating swarms, pinpointing that ecological and environmental factors might play a key role in the establishment of Wolbachia infections in the An. gambiae host [8].

Similarly, Wolbachia has never been detected in natural populations of Ae. aegypti, a main vector of Dengue and yellow fever [9].

In the last few years, several studies have revealed the potential of Wolbachia to control mosquito-borne diseases. Indeed, it has been shown that the "forced" introduction of some strains of Wolbachia in Ae. aegypti reduces its competence in transmitting Dengue virus. The mechanisms at the basis of this reduced vectorial capacity have not been elucidated, even though an upregulation of the mosquito immune response might play a role in this phenomenon $[10,11]$. More recently, it has also been proven that Wolbachia-infected Ae aegypti, resistant to Dengue virus infection, are able to rapidly replace natural/susceptible populations, thus validating the Wolbachia-mediated population-replacement strategy proposed to control mosquito borne diseases [12].

For all these reasons, there were many attempts to infect Anopheles mosquitoes with Wolbachia, in order to export the novel symbiont-based control strategies to malaria vectors. As pointed out by Hughes \& Rasgon [13], the failure of the first attempts, coupled with the apparent lack of natural infection in natural populations of anophelines, suggests that this genus is somehow refractory to Wolbachia infection. Nevertheless, in the last few years, trans-infection of cell lines and somatic infection of adults with Wolbachia have been achieved, proving that anophelines can also be forced to harbour these bacteria [14]. Recently, a strain of the main Asian malaria vector, namely Anopheles stephensi, was stably transinfected by embryonic microinjection of the $w \mathrm{AlbB}$ Wolbachia strain derived from Aedes albopictus [15].

Asaia is a bacterium stably associated with numerous mosquito species, including several anophelines, often being the dominant microorganism of the mosquito microbiota. This acetic acid symbiont localises in the gut, salivary glands and reproductive organs of adult mosquitoes. Asaia is horizontally transmitted through an oral route during feeding both in pre-adult and adult stages and through a venereal pattern during mating in adults [16, 17]. Moreover, Asaia is vertically transmitted from mother to progeny indicating that it may rapidly spread in natural mosquito populations $[17,18]$.

Both Wolbachia and Asaia have been proposed as promising microorganisms for the development of symbiont-based control methods to contrast vectorborne diseases $[3,19]$.

Here we show that the abundances of Wolbachia and Asaia are negatively related in the reproductive organs of several mosquito species, with a pattern that approximate a mutual exclusion, particularly in anophelines. This provides a possible explanation for the absence of Wolbachia in natural populations of Anopheles. For simplicity, the phenomenon investigated in this work, i.e. the interference between the two symbionts for the colonization of the mosquito gonad niche, will be referred to as 'competition'.

\section{Methods}

\section{Mosquito strains}

Insectary reared strains: The An. stephensi colony (Liston strain) has been maintained for 4 years in the insectary at the University of Camerino (Unicam). Insects were reared at $29{ }^{\circ} \mathrm{C}$ and $85-90 \%$ relative humidity with photoperiods (12:12 Light-dark). Adult insects were maintained in a $5 \%$ sucrose solution, and adult females were fed with mouse blood for egg laying. All animal experiments were carried out according to the Italian Directive 116 of 10/27/92 on the "use and protection of laboratory animals" and in adherence with the European regulation (86/609) of $11 / 24 / 86$, licence no. 125/94A, issued by the Italian Ministry of Health. The experiments were approved by the ethic committee of the University of Camerino (Protocol number 2/2014). 
Larvae were maintained in spring water and fed daily with commercial fish food.

The An. gambiae colony was established from samples obtained from the Centre National de Recherche et de Formation sur le Paludisme (CNRFP) in Ouagadougou (Burkina Faso, West Africa) and descend from a wild colony.

The Ae. albopictus colony was established from mosquitoes that were field-collected from S. Benedetto del Tronto, Central Italy (42 $\left.57^{\prime} 00^{\prime \prime} \mathrm{N} ; 13^{\circ} 53^{\prime} 00^{\prime \prime} \mathrm{E}\right)$ in 2008. They were maintained in the Unicam insectary in the same condition as described above.

Cx. quinquefasciatus species mosquitoes, collected in Hawaii (U.S.A.) in 2008 and provided by the insectary of the Center for Vector Biology, Rutgers University (New Brunswick, NJ, U.S.A.), were maintained in the Unicam insectary in the same condition described above.

The colony of An. stephensi stably trans-infected with wAlbB Wolbachia strain (and the relative control treated with antibiotics to remove Wolbachia infection) is the one described in [15], that has been bred for two generations at Unicam insectary before performing the analysis.

The colony of Ae. aegypti, stably trans-infected with $w$ MelPop Wolbachia strain (and the relative control treated with antibiotics to remove Wolbachia infection), is the one described in [20], that has been bred for two generations at Unicam insectary before performing the analysis.

Field collected mosquitoes: samples of the species $A e$. albopictus and $C x$. pipiens were collected in the following towns in the Umbria region (Central Italy): Bastia Umbra (430 $07^{\prime} 71^{\prime \prime} \mathrm{N}$; $\left.12^{\circ} 55^{\prime} 51^{\prime \prime} \mathrm{E}\right)$; Spello $\left(43^{\circ} 0^{\prime} 3^{\prime \prime} \mathrm{N} ; 12^{\circ}\right.$ $40^{\prime} 44^{\prime \prime} \mathrm{E}$ ); Foligno (42 $56^{\prime} 58^{\prime \prime} \mathrm{N}$; $12^{\circ} 43^{\prime} 10^{\prime} \mathrm{E}$ ); Collestrada $\left(43^{\circ} 05^{\prime \prime} 10^{\prime \prime} \mathrm{N} ; 12^{\circ} 28^{\prime} 47^{\prime \prime} \mathrm{E}\right)$; Ponte del Campo (430. $\left.39^{\prime \prime} \mathrm{N} ; 12^{\circ} 25^{\prime} 02^{\prime \prime} \mathrm{E}\right)$.

\section{Mosquito samples}

Total DNA was extracted from whole mosquitoes and/ or organs (dissected in a drop of sterile $1 \times$ PBS using sterile needles under a stereomicroscope) as previously described [21].

\section{Asaia and Wolbachia detection by specific PCR}

For Asaia and Wolbachia detection specific oligonuleotides were used: Asafor/Asarev [16] and WolbF (5'- gaagataatgacggtactcac $\left.-3^{\prime}\right)$ / WolbRev2 (5' - gtcagatttgaaccagataga $\left.-3^{\prime}\right)$, respectively. PCR was performed in $25-\mathrm{ml}$ reaction, using Dream taq Buffer 1X, dNTPs $0.25 \mathrm{mM}$, Asafor and Asarev oligos (0.3 mM each), 0.75U DreamTaq Polymerase (Thermo Scientific, Waltham, Massachusetts, USA) and $30 \mathrm{ng}$ of DNA template, measured with a NanoDrop ND-1000 spectrophotometer (Thermo Scientific, Waltham, Massachusetts, USA). An initial denaturation at $94{ }^{\circ} \mathrm{C}$ for 3 min was followed by 30 cycles consisting of denaturation at $94{ }^{\circ} \mathrm{C}$ for $30 \mathrm{sec}$, annealing at $60{ }^{\circ} \mathrm{C}$ for $30 \mathrm{sec}$, and extension at $72{ }^{\circ} \mathrm{C}$ for $30 \mathrm{sec}$, concluding with a final extension step of $10 \mathrm{~min}$ at $72{ }^{\circ} \mathrm{C}$. The PCR products were electrophoresed on a $1 \%$ agarose gel to determine the presence and general size of the amplified DNA.

\section{Quantitative PCR (qPCR) detection of native Asaia in different mosquito species}

PCR assays were designed to detect DNA of bacteria Asaia in organs from 13-day-old An. stephensi, Ae. albopictus and $C x$. quinquefasciatus mosquitoes. genes of the different mosquito species were amplified as housekeeping genes (As-rps7, Ae-rps7, CX-rps3 respectively) to allow the normalization of Asaia amount.

Amplification consisted of 50 ng DNA, 1X SybrGreen Master Mix (Fermentas, Vilnius, Lithuania), $200 \mathrm{nM}$ of primers. Primers used to amplify target sequences of Asaia 16S rRNA and rps7 of An. stephensi (As-rps7) were described in [22], whereas the primers for Cx-rps3 (Cx. quinquefasciatus) and Ae-rps7 (Ae. albopictus) genes are listed below:

\section{Ae-rps7-F: 5' - CGCGCTCGTGAGATCGA-3' \\ Ae-rps7-R: 5' - GCACCGGGACGTAGATCA-3' \\ Cx-rps3-F: 5' - AGCGTGCCAAGTCGATGAG-3' \\ Cx-rps3-R: 5' - ACGTACTCGTTGCACGGATCTC-3'}

Reactions were run on a CFX thermocycler (BioRad, Hercules, California, USA) using the following cycling conditions: 1 cycle of $95{ }^{\circ} \mathrm{C}$ for $10 \mathrm{~min}, 40-$ cycles of $95{ }^{\circ} \mathrm{C}$ for $1 \mathrm{~min}, 60{ }^{\circ} \mathrm{C}$ for $1 \mathrm{~min}$, and $74{ }^{\circ} \mathrm{C}$ for $30 \mathrm{sec}$. The PCRs were performed on six pools of organs, from ten individuals, for each mosquito species. Each pool was tested in duplicate. The relative quantity of Asaia in the mosquito organs was estimated as gene copy ratio calculating the copy number of Asaia 16S rRNA gene/respective housekeeping genes.

The amount of amplified targets was measured using standard curves obtained by eight serial dilutions of specific plasmids for each amplicon (from 2 to $\left.2 \times 10^{-7} \mathrm{ng}\right)$. Standard curves used in the experiments had the following parameters $\left(\mathrm{E}=\right.$ efficiency; $\mathrm{R}^{\wedge} 2=$ correlation coefficient):

Asaia: $\mathrm{E}=97.2 \% ; \mathrm{R}^{\wedge} 2=0.995 ;$ slope $=-3.392$

As-rps7: $\mathrm{E}=96.2 \% ; \mathrm{R}^{\wedge} 2=0.999 ;$ slope $=-3.416$

Ae-rps7: $\mathrm{E}=99.6 \% ; \mathrm{R}^{\wedge} 2=1.000 ;$ slope $=-3.333$

Cx-rps3: $\mathrm{E}=98.6 \% ; \mathrm{R}^{\wedge} 2=0.999 ;$ slope $=-3.356$.

\section{Statistical analysis}

The statistical analysis of the amounts of Asaia, quantified by qPCR assay, was estimated using the BioRad CFX Manager Software and the GraphPad software (http://www.graphpad.com). Data were obtained from 
the average of six pools per organ. The value of each pool resulted from the average of two technical replicates that have been compared by the Mann-Whitney test.

\section{Asaia localization on eggs-surface}

An. stephensi, An. gambiae and Ae. albopictus eggs were fixed with $4 \%$ paraformaldehyde for $10 \mathrm{~min}$ at $4{ }^{\circ} \mathrm{C}$ and washed twice with PBS $1 \mathrm{X}$. The slides were incubated in $1 \%$ Bovine Serum Albumine (BSA) in $1 \mathrm{X}$ PBS for $30 \mathrm{~min}$ at room temperature and successively for $1 \mathrm{~h}$ at $37{ }^{\circ} \mathrm{C}$ with anti-Asaia monoclonal antibody (patent pending N. MI2012A001529) diluted 1:1000. After three washings in PBS 1X, they were incubated for $30 \mathrm{~min}$ at $37{ }^{\circ} \mathrm{C}$ with anti-mouse IgG Alexa Fluor 594 conjugate (Invitrogen, Carlsbard, California, USA) diluted 1:100 in $1 \%$ BSA in PBS and washed three times for $10 \mathrm{~min}$ with $1 \mathrm{X}$ PBS. Slides were mounted with glycerol and visualized by epifluorescent microscopy (Carl Zeiss Axio Observer.Z1, Milan, Italy).

\section{Asaia and Wolbachia localization in Ae. aegypti by FISH}

Midguts and reproductive organs (from female and male mosquitoes) of Wolbachia-free and Wolbachia trans infected Ae. aegypti were dissected and fixed in $4 \%$ paraformaldehyde for $5 \mathrm{~min}$ at $4{ }^{\circ} \mathrm{C}$ and washed in $1 \mathrm{X}$ PBS. The organs were then incubated for $10 \mathrm{~min}$ at $37{ }^{\circ} \mathrm{C}$ with a $10 \mathrm{mg} \mathrm{ml}^{-1}$ pepsin solution and washed twice in a $1 \mathrm{X}$ PBS and Tween $201 \%$ solution and twice in $1 \mathrm{X}$ PBS for $5 \mathrm{~min}$ at room temperature. Hybridization was performed in dark conditions for $3 \mathrm{~h}$ at $40{ }^{\circ} \mathrm{C}$, with $100 \mu \mathrm{l}$ of hybridization buffer $(0.2 \mathrm{X}$ SSC, $40 \%$ formamide, BSA $0.1 \mathrm{mg} \mathrm{ml}^{-1}$, salmon sperm $0.1 \mathrm{mg} \mathrm{ml}^{-1}, 10 \mathrm{ng}^{-1} \mathrm{l}^{-1}$ of each probes). The probes targeting the $16 \mathrm{~S}$ rRNA gene, were synthesized by Eurofins MWG Operon (Ebersberg, Germany) and consisted of: two Asaia probes namely Asaia1.FCy3 (5' -GTGTAAACCGCCTACGCGCC-3') and Asaia2.FCy3 (5'-ATGGATAGATCCCTACGCGA-3') [17] 5'-end labeled with Cy3 (absorption/emission at 550/570 nm), and two Wolbachia probes namely W2 (5'-CTTCTGTGAGTACC GTCATTATC-3') [23] and WOL3 (5'- GATTGAAAGA GGATAGAGGA-3') [24] 5'-end labeled with Cy5 (absorption/emission at 650/670 nm). The probes specifically targeting Asaia or Wolbachia are non cross-reacting with each other.

After hybridization, the organs were washed in $200 \mu \mathrm{l}$ of washing buffer (0.2X SSC, $60 \%$ formamide) for $15 \mathrm{~min}$ at $40{ }^{\circ} \mathrm{C}$, and subsequently once in $500 \mu \mathrm{l}$ of $0.1 \mathrm{X} \mathrm{SSC}$ and twice in $200 \mu \mathrm{l}$ of $1 \mathrm{X} \mathrm{SSC}$ for $10 \mathrm{~min}$ at room temperature. After that they were washed twice in 1X PBS for $5 \mathrm{~min}$ at room temperature. Then $50 \mathrm{ng}$ of DAPI (4', 6'-diamidino-2-phenylindole) were added, and incubated for $5 \mathrm{~min}$ at room temperature. After a wash in $1 \mathrm{X}$ PBS for $5 \mathrm{~min}$ at room temperature, samples were mounted in anti-fading medium and then observed at a laser-scanning confocal microscope SP2-AOBS (Leica, Wetzlar, Germany). Control experiments involved either treatment of slides with RNase prior probe hybridization or in absence of probe.

\section{Asaia-GFP colonization of reproductive organs of different mosquito species}

An. stephensi, Cx. quinquefasciatus and Ae. albopictus mosquitoes were colonized with Asaia SF 2.1 (transformed with pHM2-Gfp plasmid) strain isolated from An. stephensi [16]. Ae. albopictus mosquitoes were also colonized with Asaia AA 5.5 (transformed with pHM2Gfp plasmid) isolated from Ae. albopictus Unicam strain.

The mosquitoes were fed with a strain of Asaia expressing the Green Fluorescent Protein (namely Asaia SF2.1 and AA5.5). These bacteria were grown $24 \mathrm{~h}$ at $30{ }^{\circ} \mathrm{C}$ in GLY medium (glycerol $25 \mathrm{~g} / \mathrm{L}$; yeast extract $10 \mathrm{~g} / \mathrm{L} ; \mathrm{pH}$ 5). Cells were harvested by centrifugation, washed three times in $0.9 \% \mathrm{NaCl}$ and adjusted to $10^{8}$ cells per $\mathrm{ml}^{-1}$ in $50 \mathrm{ml}$ of $\mathrm{H}_{2} \mathrm{O} / 5 \%$ (wt/vol) sucrose solution, supplemented with $100 \mu \mathrm{g} \cdot \mathrm{ml}^{-1}$ of kanamycin to avoid the loss of plasmid from bacterial cells. After 4 days of feeding, the cotton pad containing strain SF2.1(Gfp) was removed and replaced with a new sterile $5 \%(\mathrm{wt} / \mathrm{vol})$ sucrose solution supplemented with kanamycin. Mosquitoes were sampled and dissected every $2-3$ days up to 15 days after initial exposure to the bacterium. Guts and reproductive organs were fixed with $4 \%$ paraformaldeyde for $10 \mathrm{~min}$ at $4{ }^{\circ} \mathrm{C}$. The slides were then mounted in glycerol and examined in fluorescent microscopy (Carl Zeiss Axio Observer.Z1, Milan, Italy).

\section{Results}

\section{Tissue localization of Asaia in different mosquito species}

Firstly, by specific PCR assays we have investigated the tissue distribution of Asaia in laboratory-reared strains of Ae. albopictus and Cx. quinquefasciatus, two species of mosquitoes that are naturally infected with Wolbachia. Interestingly, while Asaia was found in the midguts of these mosquitoes, it was never detected in the reproductive organs (Table 1). This represents a remarkable difference if compared with Asaia distribution in tissues of mosquito species that are not infected with Wolbachia, such as An. gambiae, An. stephensi, Ae. aegypti, where Asaia has been constantly found in the reproductive organs and salivary glands, in addition to the midgut [25]. We have also evaluated the circulation of Asaia in different mosquito organs of An. stephensi, Ae. albopictus and $C x$. quinquefasciatus by quantitative PCR. As shown in Fig. 1, occasionally, it was possible to detect the presence of Asaia in the reproductive organs of the two latter species, but in a very limited amount compared with that present in the midguts and in the 
Table 1 PCR detection of Asaia in lab-reared mosquito species. Six pools of different organs from ten individuals per each mosquito species were analysed by Asaia-specific PCR assay

\begin{tabular}{llllll}
\hline & Anopheles gambiae & An. stephensi & Aedes aegypti & Ae. albopictus & Culex quinquefasciatus \\
\hline Male Guts & $100 \%$ Positive & $100 \%$ Positive & $100 \%$ Positive & $100 \%$ Positive & $75 \%$ Positive \\
Female Guts & $100 \%$ Positive & $100 \%$ Positive & $100 \%$ Positive & $100 \%$ Positive & $75 \%$ Positive \\
Male Gonads & $100 \%$ Positive & $100 \%$ Positive & $100 \%$ Positive & Negative & $50 \%$ Positive \\
Female Gonads & $100 \%$ Positive & $100 \%$ Positive & $100 \%$ Positive & Negative & Negative \\
\hline
\end{tabular}

reproductive organs of $A n$. stephensi. Indeed, the differences in the Asaia load in the midguts and gonads of Ae. albopictus and $C x$. quinquefasciatus, as revealed by qPCR, were evident and statistically significant. Of course, we cannot rule out the possibility that, when dissecting organs for the molecular analysis, Asaia bacterial cells located in the guts have contaminated the reproductive organs.

To further corroborate the null or very limited circulation of Asaia in the reproductive organs of Wolbachia infected mosquitoes, we have analysed Ae. albopictus and $C x$. pipiens mosquitoes field collected in Italy. A high proportion of these mosquitoes did not host Asaia (63\% and $85 \%$ respectively), as revealed by specific PCR assay on whole mosquitoes (Additional file 1: Table A).

\section{Asaia localization on eggs-surface}

The specific localization of Asaia in different tissues of mosquitoes Ae. albopictus and Cx. quinquefasciatus may explain the inability of these mosquitoes to vertically transmit Asaia, differently to what has been shown in An. gambiae [17]. To further investigate this aspect, we used a specific antibody to localize Asaia on the surface of eggs produced by different mosquito species, some naturally hosting Wolbachia and some not. Antibodybased staining revealed the presence of Asaia on the surface of eggs of An. stephensi and Ae. aegypti, while no signal was detected on the eggs of Ae. albopictus and
Cx. quinquefasciatus (Fig. 2). The results of these experiments are congruent with those of tissue localization, reinforcing the hypothesis that the presence of Wolbachia prevents the establishment of Asaia within the reproductive organs of Ae. albopictus and Cx. quinquefasciatus (and vice-versa in anopheline mosquitoes and Ae. aegypti).

\section{Asaia-GFP colonization of midguts and reproductive organs of different mosquito species}

To further investigate the hypothesis of a microbial competition between Asaia and Wolbachia within the reproductive organs of mosquitoes, a series of experiments has been performed using a strain of Asaia expressing the green fluorescent protein (GFP), provided to the mosquitoes with sugar meal to obtain body colonization. We focused our attention on midguts and gonads. In Ae. albopictus and Cx. quinquefasciatus, the colonization with Asaia-GFP strain was limited to the midguts of the examined specimens, that were colonized in the $81 \%$ and $80,3 \%$ of the cases respectively, while no colonization was detected in the reproductive organs in any of the 60 specimens analyzed for each species (Fig. 3), (Additional file 2: Table B). These findings are just the opposite with those previously reported in An. gambiae, An. stephensi and Ae. aegypti where the colonization has been repeatedly observed in both midguts and reproductive organs [3] and confirmed by the same analysis in 60 specimens An. stephensi mosquitoes, that has been

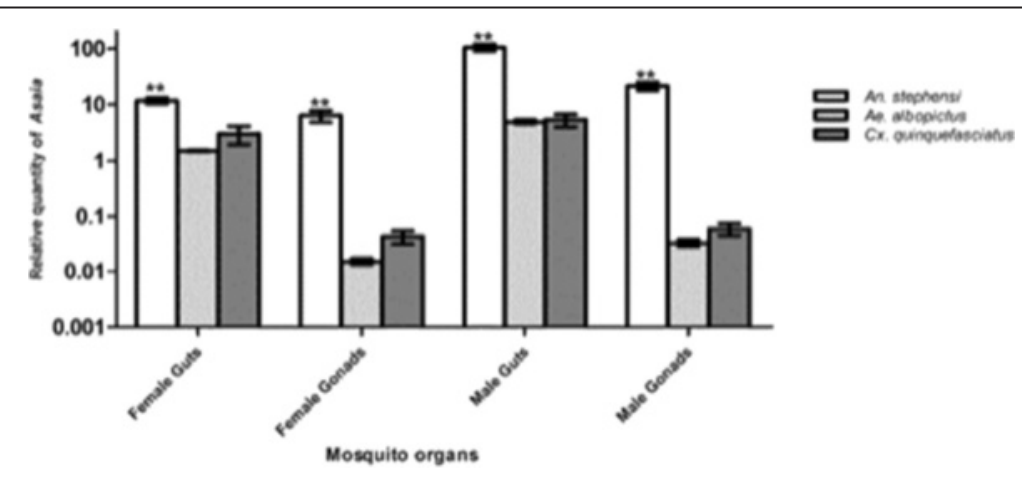

Fig. 1 Quantitative detection of Asaia in organs of three different lab-reared mosquito species obtained by qPCR. The relative amount of the bacteria is expressed as a ratio of bacterial $16 \mathrm{~S}$ rRNA and mosquito rps7 genes (An. stephensi and Ae. albopictus) or rps3 gene (CX. quinquefasciatus) copies in a logarithmic scale. Abundance results from the mean \pm SEM of six pools (10 organs) for each species. Statistically significant differences are represented by asterisks $(p<0.01)$ as determined by multiple comparisons using Mann Whitney test 


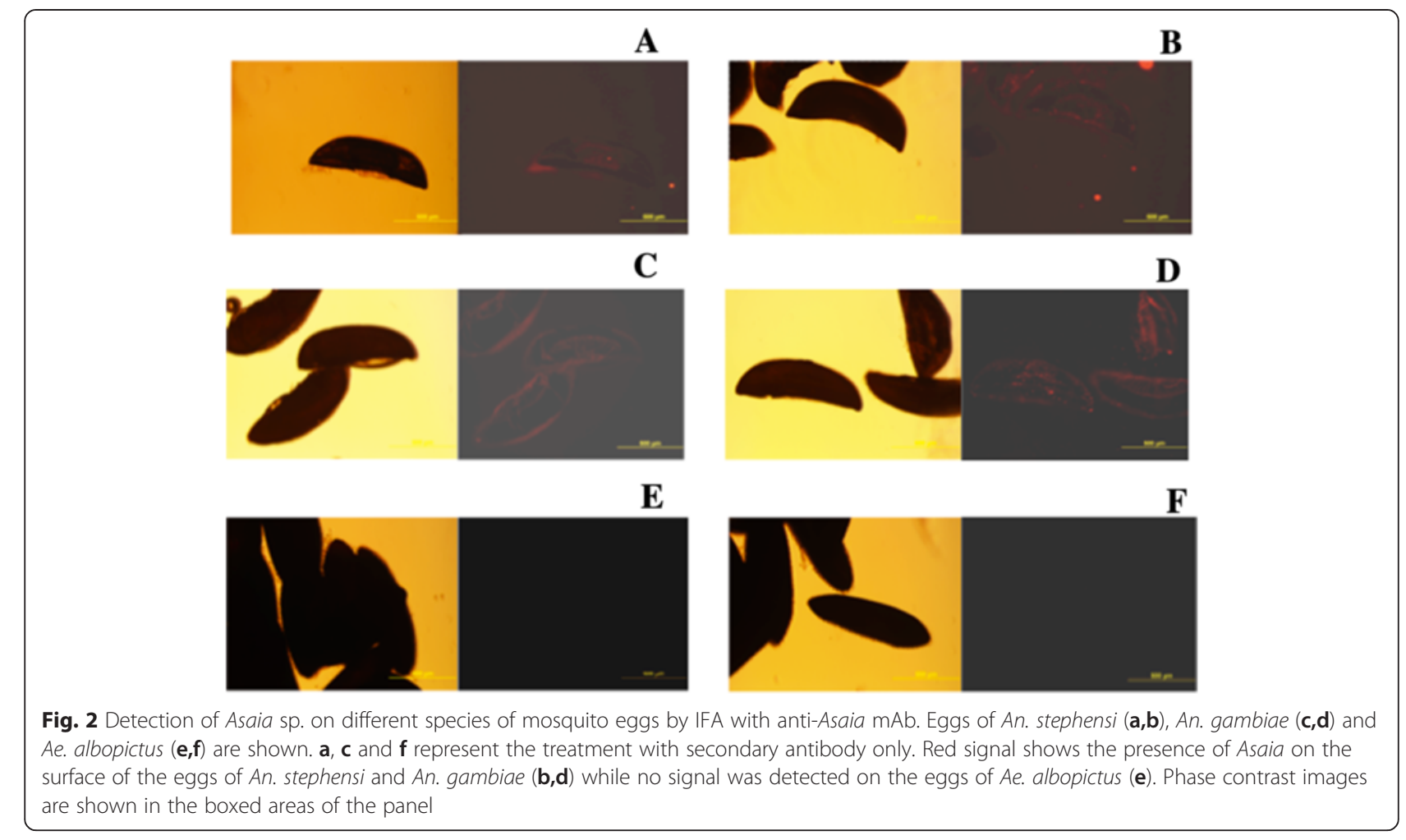

used as control, that were colonized in $86 \%$ of the guts and in $30 \%$ of the reproductive organs (Additional file 2: Table B).

Asaia-GFP colonization of reproductive organs of a strain of Ae. aegypti stably trans-infected with Wolbachia wMelPop

The colonization experiments with Asaia-GFP were then extended to a strain of Ae. aegypti stably trans-infected with a Wolbachia from Drosophila melanogaster, named wMelPop [26]. In these Wolbachia-infected Ae. aegypti mosquitoes, the Asaia colonization rate was similar to that of the control, represented by the wild type Wolbachia-uninfected Ae. aegypti from the same strain (100\% vs $100 \%$ in the guts, $36 \%$ vs $39 \%$ in the reproductive organs) (Additional file 3: Table C). Even though, these data do not seem to support a competition between the two microbes, the trans-infected strain and the control strain of Ae. aegypti have also been analysed by FISH assay to quantify the rate of infection. By comparing the intensity of the signal in wMelPop infected and uninfected mosquitoes, it was fairly evident that in the presence of Wolbachia, the amount of Asaia in the reproductive organs was lower than that in the absence of Wolbachia (Fig. 4).

\section{Asaia-GFP colonization of reproductive organs of a strain} of An. stephensi stably trans-infected with Wolbachia wAlbB The colonization experiments were extended also to a strain of An. stephensi that has been trans-infected with a Wolbachia strain from Ae albopictus, named wAlbB. The control was represented by the wild type Wolbachiauninfected strain of An. stephensi. In both the Wolbachia infected and uninfected mosquitoes the guts were constantly colonised by Asaia (100\% in both strains), while in the reproductive organs no colonization was detected in Wolbachia-positive mosquitoes. On the other hand, colonization was observed in the Wolbachia uninfected mosquitoes, in $76 \%$ of the ovaries and $20 \%$ of the testis, out of 25 specimens examined per each organ (Table 2).

\section{Discussion}

Despite its common presence in arthropods, including several mosquito species, Wolbachia has not been detected in anophelines, with just one recent description in a few mating swarms from a small population of An. gambiae [8]. To verify possible causes of this phenomenon, we have tested the hypothesis that microbial competition may contribute to explain the absence of Wolbachia infection within members of the Anopheles genus. In particular, we have tested the competition that may occur at the reproductive organs, since Wolbachia is a well-known manipulator of host reproduction that exploits effects exerted at the level of the gonads to increase its own fitness $[27,28]$. Our hypothesis is that microorganisms that should in some way reduce the amount of Wolbachia within the gonads would also reduce the overall capacity of Wolbachia to induce reproductive manipulations, such as cytoplasmic 


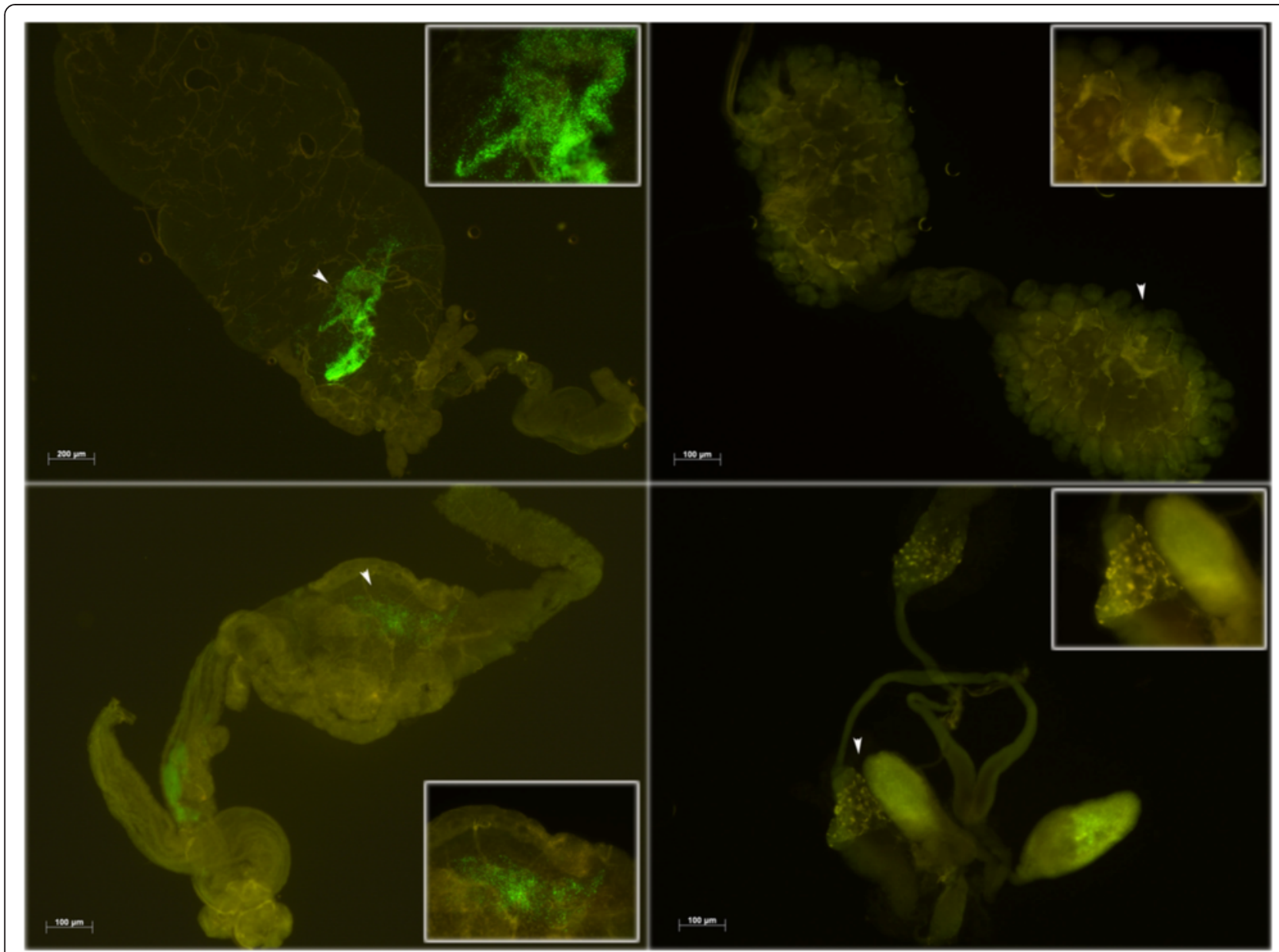

Fig. 3 Asaia-GFP colonization in different organs of Cx. quinquefasciatus. Left images show guts of female (top) and male (bottom) Cx. quinquefasciatus mosquitoes analyzed after colonization with Asaia-GFP provided with sugar solution. Arrows indicate the localization of main colonization (this area has been magnified in the square). Right images show gonads of female (top) and male (bottom) mosquitoes analyzed after colonization with Asaia-GFP. Even in the magnified area no signal of colonization is detected

incompatibility $(\mathrm{CI})$, thus reducing the capacity of Wolbachia to spread (or to be maintained) into a population.

We have focused our attention on Asaia, the only other bacterium known to localize in the mosquito's reproductive organs, and thus a potential competitor of Wolbachia for these anatomical niches.

Our overall results support the hypothesis that a competition between the two bacteria occurs at the reproductive organs, and this competition is particularly evident in the ovaries, that are essential not only for the maternal vertical transmission of Wolbachia, but also for determining phenomena like the rescuing of the sperm in $\mathrm{CI}$, and thus for ensuring the differential fitness of Wolbachia infected VS uninfected females.

First, we have shown that in Cx. quinquefasciatus and Ae. albopictus (that are naturally infected by Wolbachia), Asaia infects the gut, but does not infect the reproductive organs. Our previous works, however, showed that mosquito-species naturally uninfected with Wolbachia (i.e. An. gambiae, An. stephensi, Ae. aegypti) host Asaia in the reproductive organs, as well as in other anatomical districts $[17,29,30]$.

Secondly, the IFA-based comparative investigation, aimed at detecting the presence of Asaia on the eggs surface of An. gambiae, An. stephensi and Ae. albopictus, showed that only this latter species, the only one naturally infected with Wolbachia, did not carry Asaia on the egg surface. This is congruent with the absence of Asaia in the reproductive organs in Ae. albopictus, which is thus to be regarded as a dead-end host for these bacteria, in contrast to what was observed in An. stephensi (this study) and An. gambiae [17], where egg-smearing appears as the mechanism for the vertical transmission of these bacteria.

Thirdly, the colonization with an Asaia-GFP strain was detectable only in the guts, and not in the gonads, in Cx. quinquefasciatus and Ae. albopictus, while this strain of Asaia has already been shown to be very 


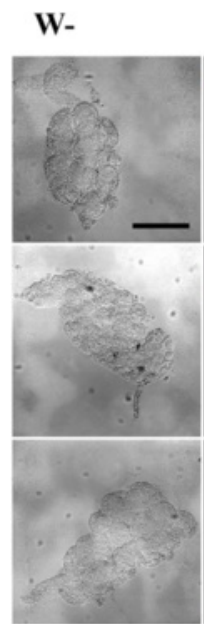

1

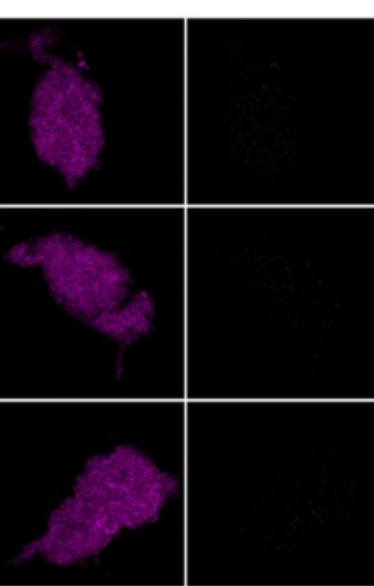

2

3

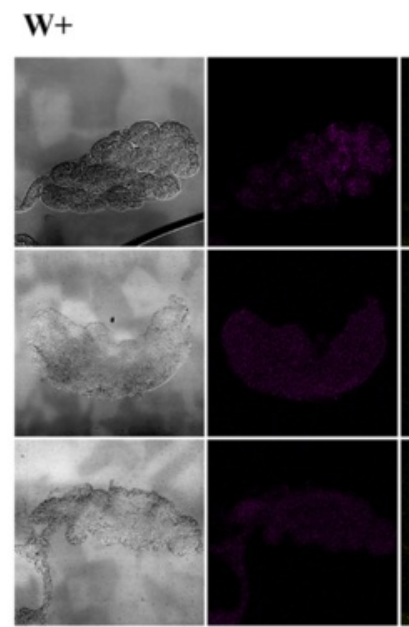

1

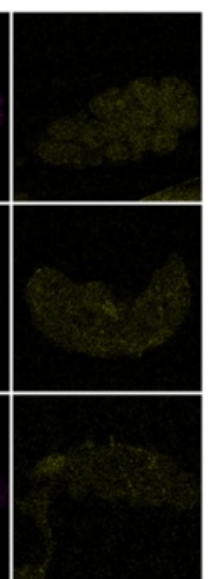

3

Fig. 4 Asaia and Wolbachia detection in gonads of wMelPop infected (W+) and uninfected (W') Ae. aegypti mosquitoes. Detection is shown through interferential contrast microscopy (column 1) and after whole mount in situ hybridization with Asaia (in magenta, column 2) and Wolbachia (in yellow, column 3) specific probes. Bar corresponds to 120 um

effective in the colonization of the reproductive organs in An. gambiae, An. stephensi, Ae. aegypti [3, 25].

Finally, we showed that Asaia-GFP is able to colonize two Wolbachia artificially and stably infected strains of mosquito (Ae. aegypti and An. stephensi). In both cases we observed that an unnatural presence of Wolbachia in guts and reproductive organs does not alter the ability of Asaia-GFP to efficiently colonize the guts of recipient mosquitoes. Conversely, the presence of Wolbachia in the reproductive organs may strongly influence the ability of Asaia-GFP to efficiently colonize the reproductive organs of trans-infected mosquitoes in respect to the control strains. In Ae. aegypti we detected a similar percentage of colonized mosquitoes between Wolbachia infected and uninfected mosquitoes, although Asaia has been shown to be less abundant in the reproductive organs of Wolbachia-positive mosquitoes, compared to Wolbachia-negative ones.

In An. stephensi this difference between Wolbachiapositive and -negative mosquitoes was stronger, in that

Table 2 Colonization experiments with Asaia-GFP of a strain of Anopheles stephensi stably trans-infected with Wolbachia (W+) and a wild type Wolbachia-uninfected An. stephensi strain $\left(\mathrm{W}^{-}\right)$. Asaia-GFP was provided to mosquitoes with the sugar meal. Twenty five organs were analysed per each strain and relative positivity is reported

\begin{tabular}{|c|c|c|}
\hline & An. stephensi W+ & An. stephensi W \\
\hline Guts ô & $100 \%$ & $100 \%$ \\
\hline Guts ㅇ & $100 \%$ & $100 \%$ \\
\hline Reproductive organs $\widehat{\partial}$ & $0 \%$ & $20 \%$ \\
\hline Reproductive organs $q$ & $0 \%$ & $76 \%$ \\
\hline
\end{tabular}

no colonization was observed in the reproductive organs from Wolbachia-positive mosquitoes, while colonization of reproductive organs in Wolbachia-negative insects was detected in about $75 \%$ of the specimens tested. The above reported differences between Ae. aegypti and An. stephensi may be due to intrinsic species-specific factors and/or to the fact that the two species were infected with different Wolbachia strains: Ae. aegypti with one from Drosophila, the virulent strain wMelPop of Wolbachia, and An. stephensi with one from Ae. albopictus.

All these evidences taken together, underline a role of Wolbachia in preventing some mosquito species from a stable and successful Asaia infection in the gonads. On the other hand, our data seem to support a role of Asaia in the inability of Wolbachia to infect anopheline mosquitoes.

Asaia seems to exert its action specifically at the level of reproductive organs and particularly in ovaries suggesting that these two bacteria may compete for the same anatomical niche or infection routes in the host reproductive organs so that in the presence of Asaia, Wolbachia cannot colonize these organs, or that colonization is in some way limited. As already emphasized, a limited colonization of male and female gonads is likely to reduce the strength of reproductive manipulations such as $\mathrm{CI}$, and, in the case of the female gonad, a limited (or absent) colonization will obviously reduce the efficacy of vertical transmission.

These conclusions are congruent with the results of a recent study described by Hughes and collaborators that have shown that Asaia may impede vertical transmission of $\mathrm{Wol}$ bachia in Anopheles. [31]. Conversely, it has recently been demonstrated also that the introduction of $w \mathrm{AlbB}$ in An. stephensi reduces female fecundity and causes a minor decrease in male mating competiveness [32]. 


\section{Conclusions}

Symbiont competition and in particular the competition for anatomical niches such as the gonads appears an important but under-investigated phenomenon, that is likely to have an impact on the establishment of symbioses in insects.

Although we cannot exclude that other microorganisms may take part in the "competition for gonads", it is reasonable to assume that the phenomenon is not necessarily based on a 'complete absence' VS a 'complete presence' of particular symbionts. Nevertheless, our findings update the current knowledge on mosquito symbiosis and may have important implications for the development of symbiont-based control of mosquito-borne diseases, a research area where both Asaia and Wolbachia are regarded as important candidates.

\section{Additional files}

Additional file 1: Table A. Assessment of Asaia and Wolbachia circulation in field collected mosquitoes. Percentage of field collected mosquitoes naturally infected with Asaia and Wolbachia was detected by specific PCR assay. The total number of mosquito examined was 104.

Additional file 2: Table B. Asaia-GFP tissue specific colonization. Sixty individuals of three different mosquito species were provided with sugar meal enriched with Asaia-GFP. Percentages of colonized guts and gonads are reported.

Additional file 3: Table C. Colonization experiments with Asaia-GFP in a strain of Ae. aegypti stably trans-infected with a Wolbachia (W+) and wild type Wolbachia-uninfected Ae. aegypti strain $\left(\mathrm{W}^{-}\right)$. Sixty individuals of both mosquito species were provided with sugar meal enriched with Asaia-GFP. Percentages of colonized guts and gonads are reported.

\section{Competing interests}

The authors declare that they have no competing interests.

\section{Authors' contributions}

GF conceived the study and contributed to it with material collection, data analysis, interpretation and manuscript writing. PR, IR, AC, CD, UU, MVM, MV, AiC, MM, PS, performed field samples, molecular analysis and colonization experiments. MM and LS performed ISH and molecular analysis. SE, EC and BC contributed to data analysis. DJ, ZX, So'N provided some strains of mosquitoes transinfected with Wolbachia and contributed to data interpretation and manuscript writing. All authors read and approved the final version of the manuscript.

\section{Acknowledgments}

G.F. wishes to thank his inspirer in the area of symbiosis, a unique "sarchiapone", Prof. Claudio "Pippo" Bandi, as well as Dr. Roberto Spurio for critical revision of the manuscript.

The research leading to these results has received funding Prin 2012 (protocol 2012T85B3R), from the Italian Ministry of Education, University and Research (MIUR) and by the EU-FP7 Capacities-Infrastructure 2008 (grant 228421) to GF and from European Union Seventh Framework Programme ([FP7/2007-2013] [FP7/2007-2011]) under grant agreement n. 281222 to IR. The funders had no role in study design, data collection and analysis, decision to publish, or preparation of the manuscript.

\section{Author details}

${ }^{1}$ Scuola di Bioscienze e Medicina Veterinaria, Università di Camerino, 62032 Camerino, MC, Italy. ${ }^{2}$ Dipartimento di Scienze Veterinarie e Sanità Pubblica, DIVET, Università degli Studi di Milano, Milan, Italy. ${ }^{3}$ Dipartimento di Scienze per gli Alimenti la Nutrizione e I'Ambiente, DeFENS, Università degli Studi di Milano, Milan, Italy. ${ }^{4}$ Department of Microbiology and Molecular Genetic,
Michigan State University, Michigan, USA. ${ }^{5}$ Dipartimento di Scienze della Vita, Università di Modena e Reggio Emilia, Modena, Italy. ${ }^{6}$ Dipartimento di Biologia e Biotecnologie "L. Spallanzani", Università di Pavia, Pavia, Italy. ${ }^{7}$ School of Biological Sciences, Monash University, Clayton, VIC, Australia.

Received: 1 September 2014 Accepted: 7 May 2015

Published online: 17 May 2015

\section{References}

1. Moya A, Peretó J, Gil R, Latorre A. Learning how to live together: genomic insights into prokaryote-animal symbioses. Nat Rev Genet. 2008;9:218-29.

2. Dale C, Moran NA. Molecular interactions between bacterial symbionts and their hosts. Cell. 2006;126:453-65.

3. Ricci I, Valzano M, Ulissi U, Epis S, Cappelli A, Favia G. Symbiotic control of mosquito borne disease. Pathog Glob Health. 2012;106:380-5.

4. Bandi C, Anderson TJ, Genchi C, Blaxter ML. Phylogeny of Wolbachia in filarial nematodes. Proc Biol Sci. 1998;265:2407-13.

5. Zug R, Hammerstein P: Bad guys turned nice? A critical assessment of Wolbachia mutualisms in arthropod hosts. Biol Rev Camb Philos Soc 2014, doi:10.1111/brv.12098.

6. Hertig M, Wolbach SB. Studies on Rickettsia-Like Micro-Organisms in Insects. J Med Res. 1924:44:329-74.

7. Ricci I, Cancrini G, Gabrielli S, D'Amelio S, Favia G. Searching for Wolbachia (Rickettsiales: Rickettsiaceae) in mosquitoes (Diptera: Culicidae): large polymerase chain reaction survey and new identifications. J Med Entomol. 2002;39:562-7.

8. Baldini F, Segata N, Pompon J, Marcenac P, Robert Shaw W, Dabiré RK, et al. Evidence of natural Wolbachia infections in field populations of Anopheles gambiae. Nat Commun. 2014;5:3985. doi:10.1038/ncomms4985.

9. Ruang-Areerate T, Kittayapong P. Wolbachia transinfection in Aedes aegypti: a potential gene driver of dengue vectors. Proc Natl Acad Sci U S A. 2006:103:12534-9.

10. Kambris Z, Cook PE, Phuc HK, Sinkins SP. Immune activation by life-shortening Wolbachia and reduced filarial competence in mosquitoes. Science. 2009;326:134-6.

11. Sinkins SP. Wolbachia and arbovirus inhibition in mosquitoes. Future Microbiol. 2013:8:1249-56.

12. Hoffmann AA, Montgomery BL, Popovici J, Iturbe-Ormaetxe I, Johnson PH, Muzzi F, et al. Successful establishment of Wolbachia in Aedes populations to suppress dengue transmission. Nature. 2011;476:454-7.

13. Hughes GL, Rasgon JL. Transinfection: a method to investigate Wolbachia-host interactions and control arthropod-borne disease. Insect Mol Biol. 2014;23:141-51.

14. Hughes GL, Pike AD, Xue P, Rasgon JL. Invasion of Wolbachia into Anopheles and other insect germlines in an ex vivo organ culture system. PLoS One. 2012; $7:$ :36277.

15. Bian G, Joshi D, Dong Y, Lu P, Zhou G, Pan X, et al. Wolbachia invades Anopheles stephensi populations and induces refractoriness to Plasmodium infection. Science. 2013;340:748-51.

16. Favia G, Ricci I, Damiani C, Raddadi N, Crotti E, Marzorati M, et al. Bacteria of the genus Asaia stably associate with Anopheles stephensi, an Asian malarial mosquito vector. Proc Natl Acad Sci U S A. 2007;104:9047-51.

17. Damiani C, Ricci I, Crotti E, Rossi P, Rizzi A, Scuppa P, et al. Mosquito-bacteria symbiosis: the case of Anopheles gambiae and Asaia. Microb Ecol. 2010;60:644-54.

18. Damiani C, Ricci I, Crotti E, Rossi P, Rizzi A, Scuppa P, et al. Paternal transmission of symbiotic bacteria in malaria vectors. Curr Biol. 2008;18:R1087-8.

19. Bourtzis K, Dobson SL, Xi Z, Rasgon JL, Calvitti M, Moreira LA, et al. Harnessing mosquito-Wolbachia symbiosis for vector and disease control. Acta Trop. 2014;132(Suppl):S150-63

20. McMeniman CJ, Lane RV, Cass BN, Fong AW, Sidhu M, Wang YF, et al. Stable introduction of a life-shortening Wolbachia infection into the mosquito Aedes aegypti. Science. 2009;323:141-4.

21. Favia G, Dimopoulos G, della Torre A, Touré YT, Coluzzi M, Louis C. Polymorphisms detected by random PCR distinguish between different chromosomal forms of Anopheles gambiae. Proc Natl Acad Sci U S A. 1994;91:10315-9.

22. Capone A, Ricci I, Damiani C, Mosca M, Rossi P, Scuppa P, et al. Interactions between Asaia, Plasmodium and Anopheles: new insights into mosquito symbiosis and implications in malaria symbiotic control. Parasit Vectors. 2013;6:182. 
23. Heddi A, Grenier AM, Khatchadourian C, Charles H, Nardon P. Four intracellular genomes direct weevil biology: nuclear, mitochondrial, principal endosymbiont, and Wolbachia. Proc Natl Acad Sci U S A. 1999;96:6814-9.

24. Sanguin $\mathrm{H}$, Herrera A, Oger-Desfeux C, Dechesne A, Simonet P, Navarro E, et al. Development and validation of a prototype $16 \mathrm{~S}$ rRNA-based taxonomic microarray for Alphaproteobacteria. Environ Microbiol. 2006;8:289-307.

25. Ricci I, Damiani C, Capone A, DeFreece C, Rossi P, Favia G. Mosquito/ microbiota interactions: from complex relationships to biotechnological perspectives. Curr Opin Microbiol. 2012;15:278-84.

26. Woolfit M, Iturbe-Ormaetxe I, Brownlie JC, Walker T, Riegler M, Seleznev A, et al. Genomic evolution of the pathogenic Wolbachia strain, wMelPop. Genome Biol Evol. 2013;5:2189-204.

27. Bordenstein SR, O'Hara FP, Werren JH. Wolbachia-induced incompatibility precedes other hybrid incompatibilities in Nasonia. Nature. 2001;409:707-10.

28. Saridaki A, Bourtzis K. Wolbachia: more than just a bug in insects genitals. Curr Opin Microbiol. 2010;13:67-72.

29. Favia G, Ricci I, Marzorati M, Negri I, Alma A, Sacchi L, et al. Bacteria of the genus Asaia: A potential weapon against malaria. Adv Exp Med Biol. 2008:627:49-59.

30. Crotti E, Damiani C, Pajoro M, Gonella E, Rizzi A, Ricci I, et al. Asaia, a versatile acetic acid bacterial symbiont, capable of cross-colonizing insects of phylogenetically distant genera and orders. Environ Microbiol. 2009;11:3252-64

31. Hughes GL, Dodson BL, Johnson RM, Murdock CC, Tsuijimoto H, Suzuki Y, et al. Native microbiome impedes vertical transmission of Wolbachia in Anopheles mosquitoes. Proc Natl Acad Sci U S A. 2014:111:12498-503.

32. Joshi D, Mc Fadden MJ, Bevins D, Zhang F, Xi Z. Wolbachia strain wAlbB confers both fitness costs and benefit on Anopheles stephensi. Parasit Vectors. 2014;7:366

\section{Submit your next manuscript to BioMed Central and take full advantage of:}

- Convenient online submission

- Thorough peer review

- No space constraints or color figure charges

- Immediate publication on acceptance

- Inclusion in PubMed, CAS, Scopus and Google Scholar

- Research which is freely available for redistribution 